\title{
Methylprednisolone Sodium Succinate
}

National Cancer Institute

\section{Source}

National Cancer Institute. Methylprednisolone Sodium Succinate. NCI Thesaurus. Code C48004.

The sodium succinate salt of a synthetic glucocorticoid receptor agonist with immunosuppressive and anti-inflammatory effects. Methylprednisolone sodium succinate is converted into active prednisolone in the body, which activates glucocorticoid receptor mediated gene expression. This includes inducing synthesis of antiinflammatory protein IkappaB-alpha and inhibiting synthesis of nuclear factor kappaB (NF-kappaB). As a result, proinflammatory cytokine production such as IL-1, IL-2 and IL-6 is down-regulated and cytotoxic T-lymphocyte activation is inhibited. Therefore, an overall reduction in chronic inflammation and autoimmune reactions may be achieved. 